\title{
Research for Building interactive environment Based on Modular Design model
}

\author{
Fan Kang \\ Wuhan Polytechnic University, Wuhan, China \\ Corresponding E-mail:Kangfaer22@126.com
}

Keywords: Modular Design, Home Electronics, Network, landscape environment

\begin{abstract}
In this paper, we propose a modular equipment design concept for building interactive landscape environment, which can deal with the problems such as life expectancy and complicated cable networks. Recently, the advancement of digital technology in residential networking and connections (i.e., internet cables, power cables, and network cables) has made a positive alteration to the interspa-tial relationship between appliances. Moreover, the interaction and communication between user and application has understood progressive changes. In future home landscape environment, the living room is a place that has become a haven for the family and a place for entertainment as well. The down-side of the new technologies is the life expectancy of the product, which is affected by a simple change to the development of digital technology.
\end{abstract}

\section{Introduction}

As digital products have the attribute of being function-centered, they offer the possibility of fusing furniture and space within the home (Dresselhaus, 2004; Kim, 2007; Kim, 2008). These new forms of digital-conversion home electronics may greatly change our daily lives and home landscape environments. In the future, the home landscape landscape environment equipped with a digital network will not simply be a place for the family to relax, but is also expected to play a role as the final destination for the various data produced by information society, and as a place for the consumption and production of information. In other words, at the same time as being a place to relax, it will be a place for work, a place for enjoyment, and a place for learning (Newman, 1998; Weiser, 1991). Therefore, a new paradigm is required alongside the changes to products. Since the digital revolution, in conjunction with the development of information-processing equipment and home electronics, various technologies have entered into our living spaces and gradually become intelligent (Munro, 1991; Munn, 1989) . At the same time, new relation-ships need to be established between home electronics and home spaces. These changes are taking place against the backdrop of the emergence of products that blur the distinction between home electronics and furniture (Wimmer, 2001). This research looks directly at the roles of digital technology in home electronics and furniture, and proposes a new product image for the future from the fusion of home electronics, furniture, and spaces that are created from the interactions between them. The aim is to create a new conception of the home electronics landscape environment from digital technologies and culture.

\section{The Relationship between Behavior in the Living Space and Furniture}

If we exclude going to bed and housework, the majority of activities in the home space occur centered in the living room. Many of the actions in our daily lives, such as acquiring information, communication, and entertainment are accomplished in the living room. Therefore, there are expectations for the rational utilization of furniture within it; specifically, for the fusion and connection of home electronics and furniture. Within this, the technology that will be used in the homes of the future and the module home electronics that are constantly advancing need to be de-veloped into a form that can continuously respond to changes in technologies and lifestyles. In the living space.As shown in Figure 1, the appliances that are used, which are centered on the members of the family's acquisition of information, communication, and entertainment duplicate 
each other but are also different in terms of shape, size, and standards. This is the current situation, in which they are all jumbled together within a narrow space. Therefore, there is a need to propose a new integrated form of home electronics through their physical and technological fu-sion, the modularization of size, and moreover, the application of a new interface for composite devices (Show in Figure 2).

Speaker
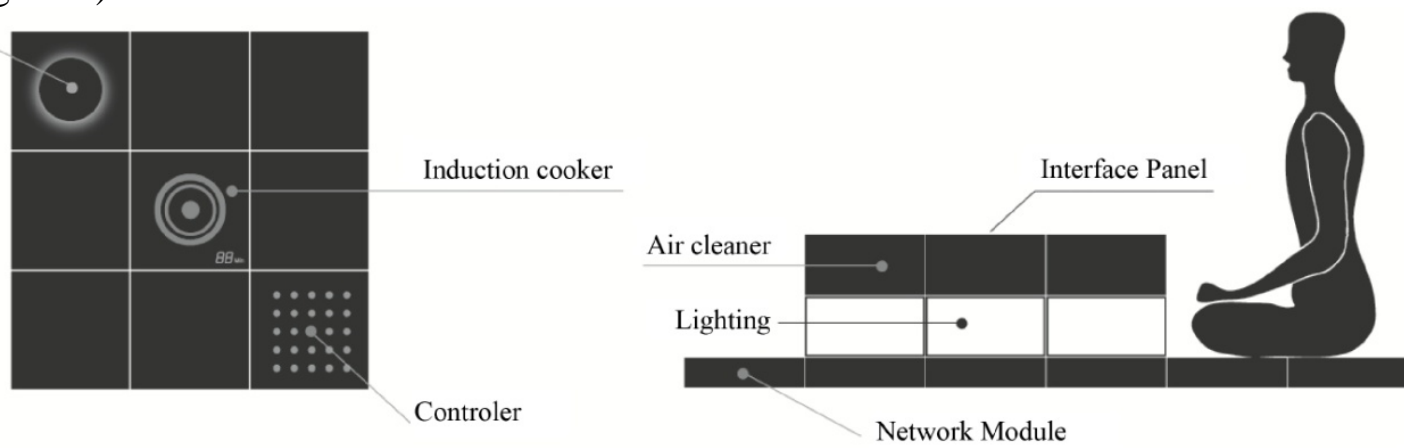

Figure 1. The module structure.
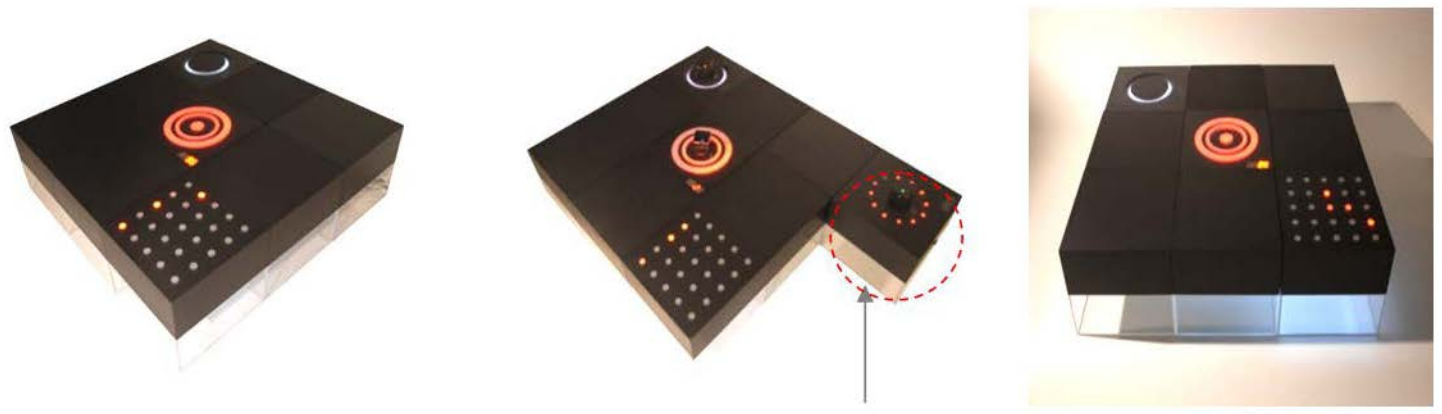

Home Server Module

Figure 2. The module combination for a communications table.

\section{Changes to the Living Space through the Fusion of Home Electronics and Furniture}

Figure 3 shows a pattern frequently seen in the layouts of existing living spaces, with the table and couch placed at the center of the living room and home electronics by the walls. A constant distance is maintained from the couch to the TV, and the various related devices are kept on the AV rack centered on the TV. In this living room, the members of the family sit on the sofa orientated in a single direction, and basically use the re-mote controls to operate the equipment. The fusion of home electronics and furniture greatly changes this ar-rangement and spatial configuration. The members of the family gather centered on the living space and come to be able to converse and enjoy entertainment while facing each other .In addition, when oper-ating home electronics, they are able to easily use a wide range of functions through an intuitive interface that utilizes their five senses. 

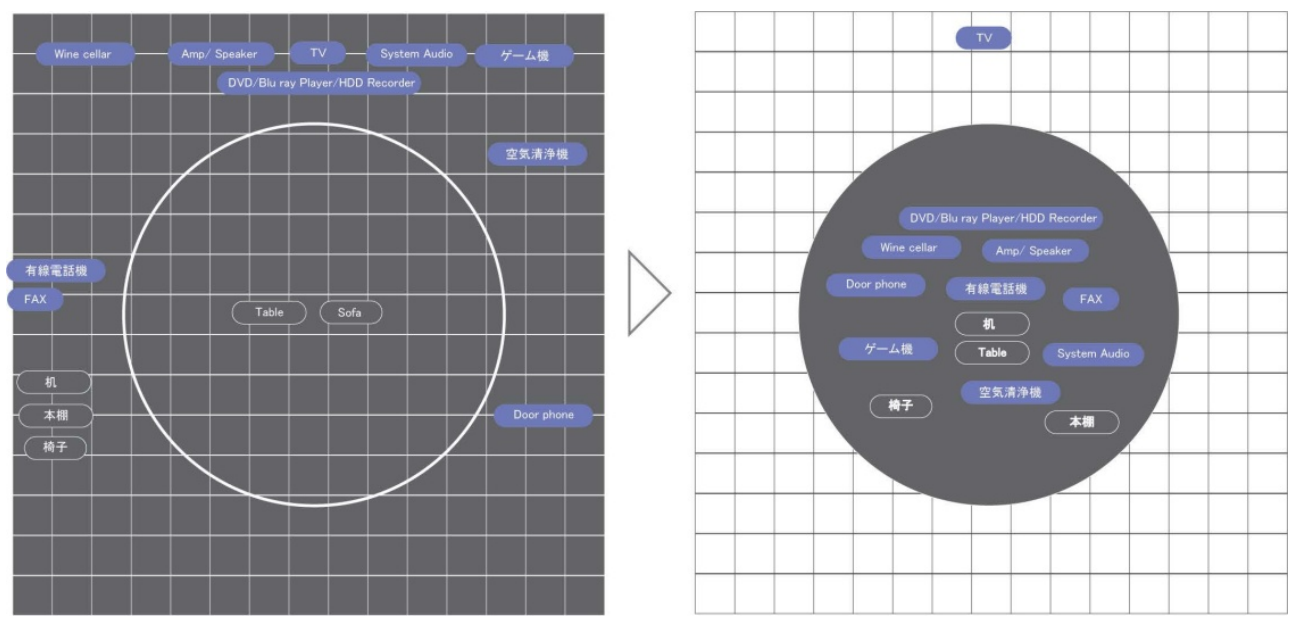

Figure 3. The existing layout of home electronics and furniture. The new layout following their fusion.

Design Principle 1 (Basic Module)

For the basic module size $(300 \times 300 \times 150)$, the focus is currently being placed on improving its feasibility further, for example its assembly and disassembly, by setting basic units of size for the distributed architectural components. In addition, focusing on the module, the greatest emphasis is being placed on a design that increases the freedom to adapt the layout of the furniture and space. The functional structure of the module was conceived to achieve composite multi-functions by installing one function in one module box, and then combining the modules. The types of installed functions are divided into categories centered on the genres of existing equipment, including AV equipment, computer-related products, and telecommunications equipment. In addition, the aim is for a module design that can be divided into a spatial element and a furniture element in accordance with a physical scale that can accommodate these devices.

Design Principle 2 (Coordination with the Furniture and the Space)

Basically, one module is equipped with one function. The module can exist as an independent unit, but it is also possible to increase its functions through connecting it with modules with other functions. Also, by connecting the modules, it is possible to not only expand the product's functions but, in addition to this, its physical function as furniture. In other words, while functioning as home electronics, they can also be used as furniture through the various combinations of modules. To take it one step farther, it is possible to apply this to walls, ceilings, pillars, floors, and the other elements that form the building. In this way, it is considered that by not restricting a module with one function to simply being physical hardware but instead expanding it into a wider space (furniture and the building), the module is able to continuously respond to changes to the landscape environment.

\section{The Module Structure}

Definition of the Function Unit

In the product structure, it incorporates the functions and is responsible for direct interface with the user. It is necessary to visually express the functions of the product, and the operation of the functions is accomplished by an internal touch sensor on the surface. Changes to the internal digital source can be upgraded via the Internet. It also functions as a display by using materials that transmit light. It must be heat resistance and durable (Figure 4). 


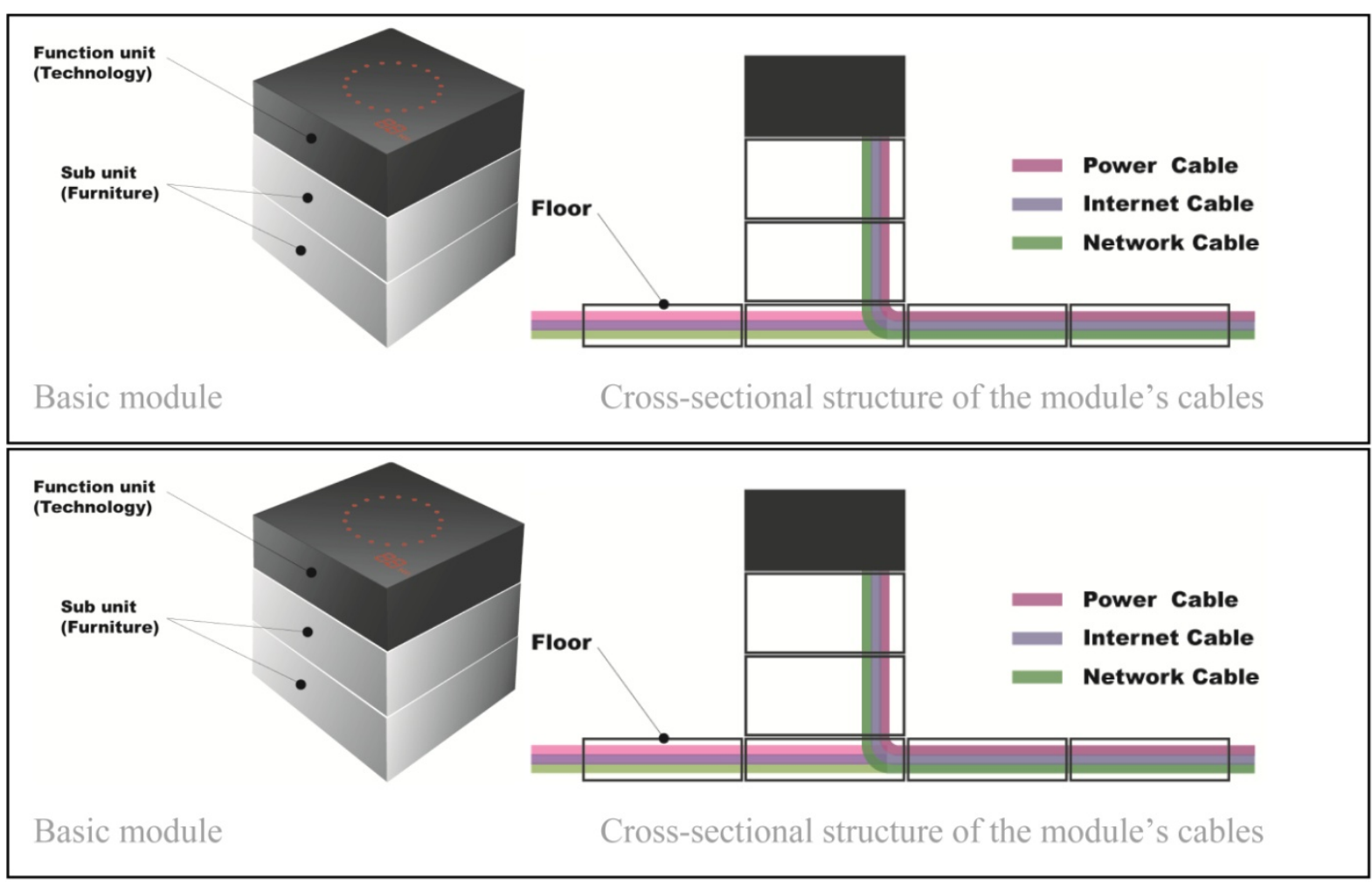

Figure 4 Basic module and cross-sectional structure of the module's cables.

\section{Conclusions}

Within living spaces made intelligent by digital technology, we must extend concepts in order to create sustain-able products that exist alongside people. Also, by introducing the medium of furniture, it becomes possible to construct new spaces or new lifestyles. The "communications table" is only one example of module home electronics described by the author. But looking toward the digital network society of the future, we can expect the concepts we have held for home electronics up to the present time to be greatly transformed. In terms of issues for the future, in order to more smoothly connect home electronics to spaces, research is required to rationalize the modules through their connective structure and to also rationalize the interface so it is able to transmit digital functions without any sense of incompatibility. In conjunction with this research, work is needed to discover and develop new materials.

\section{Acknowledgment}

This paper is funded by projects:Wuhan polytechnic university of teaching research project(XM2015021);Wuhan polytechnic university Introduced talents research initiative project (20152S05).

\section{Reference}

[1]Dresselhaus, B. (2004). Design Innovation 1.0. Portland: Dresselhaus Design Group. Kim, M. (2008). Understanding of Ubiquitous. Seoul: Ihan.

[2]Kim, N. (2007). A Study on the Modula Coordination for the interactive. Seoul.

[3]Munn, R. E. (1989). Towards Sustainable Development: An landscape environmental Perspective. Economy and Ecology: Towards Sustainable Development. New York.

[4]Munro, D. A. (1995). Sustainability: Rhetoric or Reality? In Trzyna, \& Thaddeus (Eds.). California: Center for the Envi-ronment and Public Policy. 
[5]Newman, P. (1998). Sustainability and Cities. Washington: Island Press.

[6] Weiser, M. (1991). The Computer for the 21st Century. New York: Scientific American Press. Wimmer, W. (2001). ECO Design Pilot. Massachusetts: Kluwer Academic Publishers. 